\title{
OPTIMIZACIÓN EN CONTROL VISUAL DE ROBOTS MANIPULADORES
}

\author{
Álvaro Belmonte, Jorge Pomares, Gabriel J. García \\ Departamento de Física, Ingeniería de Sistemas y Teoría de la Señal \\ Carretera San Vicente del Raspeig, s/n, San Vicente del Raspeig, CP: 03690 \\ Universidad de Alicante \\ bb35@alu.ua.es, \{jpomares; gjgg\}@ua.es
}

\begin{abstract}
Resumen
En este artículo se describe la formulación de un sistema de control visual directo basado en imagen para el seguimiento de trayectorias con optimización del esfuerzo articular. Se describen los principales componentes de esta estrategia de control para su aplicación al guiado de manipuladores. Haciendo uso de esta ley de control es posible, no sólo optimizar los pares a aplicar para el seguimiento de trayectorias, sino también balancear o especificar las articulaciones que soportarán más o menos esfuerzo durante el seguimiento. Tras realizar la formulación se demuestra la validez del mismo mediante simulación de un robot de tres grados de libertad.
\end{abstract}

Palabras clave: Control visual, control de robots, control óptimo.

\section{INTRODUCCIÓN}

Los sistemas de control visual clásicos permiten realizar el posicionamiento punto a punto de un robot haciendo uso de información visual [1]. Como se menciona en [7], hasta mediados de la década de los 90 fueron pocos los controladores propuestos basados en visión que tenían en cuenta la dinámica no lineal de los robots manipuladores. En estas dos últimas décadas la tendencia sigue siendo la misma, aunque en los últimos años se aprecia un aumento de trabajos relacionados con el control visual directo basado en imagen para vehículos autónomos no tripulados. Sin embargo, centrando la atención de nuevo en los robots manipuladores, son pocos los trabajos donde se tiene en cuenta la dinámica del robot. Es decir, la mayoría de los controladores desarrollados se diseñan bajo la asunción de que el robot es un dispositivo de posicionamiento perfecto con una dinámica despreciable, lo que reduce el problema a un problema de control cinemático basado en las imágenes adquiridas por la cámara.

El control visual directo es una técnica que permite obtener un comportamiento de alto nivel de los sistemas robóticos controlados por visión. La eliminación del lazo de control interno del robot permite un procesamiento de la imagen a una frecuencia muy elevada. Los trabajos existentes en la literatura en los que se describe el control visual directo de un robot tratan, en la mayoría de los casos, con robots con pocos grados de libertad. A principios de esta década, cabe reseñar los trabajos realizados por Cheah et al. [2][3] con el propósito de implementar un controlador visual directo adaptativo capaz de llevar a cabo la tarea correctamente ante errores en el modelo dinámico o algunos parámetros de la tarea, como la profundidad o distancia entre la cámara y el objeto observado. En [13] se propone una Jacobiana de la imagen que no depende de la distancia entre la cámara y el objeto. En general, para los controladores visuales directos, así como para los indirectos, sólo se ha demostrado su estabilidad localmente, es decir, en un entorno cercano a la configuración deseada. Sin embargo, en 2013 se publicó un trabajo que trata de conseguir una demostración de estabilidad global para los controladores visuales directos a partir del secuenciamiento de tareas [8]. En [10] se describe una estrategia de control visual directo para el seguimiento de trayectorias en la imagen, considerando que las referencias visuales en cada instante del seguimiento dependen del tiempo. Esto permite asegurar una estabilidad global a partir de una serie finita de posiciones relativas muy cercanas unas a otras (y, por lo tanto, locales).

Recientemente, Mahony propone un framework para el diseño de nuevos controladores visuales directos basados en imagen [9]. El enfoque propuesto utiliza la estructura de los sistemas hamiltonianos con puertos. Una ventaja clave de este framework es que cada característica de imagen se trata como un puerto de energía separado conectado a la dinámica del sistema. De este modo, las características de imagen separadas pueden ser tratadas como módulos desmontables en el diseño de control que conduce a una serie de ventajas. En cuanto a las aplicaciones de los sistemas de control visual dinámico basado en imagen, es habitual encontrar en los últimos tiempos trabajos relacionados con vehículos aéreos no tripulados [4][5][11][14]. También se encuentran trabajos donde se tiene en 
cuenta la dinámica de manos robóticas para definir tareas de agarre y manipulación basándose en la información visual y táctil [6].

En este artículo se presenta un framework basado en control óptimo para la implementación de sistemas de control visual directo basado en imagen para el seguimiento de trayectorias. Se describen los principales componentes de este framework y se muestran las últimas simulaciones realizadas en las que se comprueba su correcto comportamiento y la posibilidad del ajuste de su comportamiento dinámico. En la Sección 2 se describe la formulación general de un sistema de control visual basado en par calculado. Este sistema permite generar la aceleración que permite realizar el seguimiento de la trayectoria deseada en el espacio imagen. Esta información es empleada para generar los pares articulares en el caso de implementar una estrategia de control visual directo. En la Sección 3 se extienden estos controladores para la implementación de un sistema de control visual directo con optimización del esfuerzo articular durante el seguimiento. La Sección 4 describe los principales resultados obtenidos en simulación.

\section{CONTROL VISUAL BASADO EN PAR CALCULADO}

En este Apartado se describirá la formulación básica de un sistema de control visual basado en par calculado para el seguimiento de trayectorias. En la Subsección 2.1 se describe la formulación indirecta para pasar, en la Subsección 2.2, a extenderla a una estrategia de control visual directo basado en imagen.

\subsection{CONTROL VISUAL INDIRECTO BASADO EN ACELERACIÓN}

En primer lugar, se van a definir algunas cuestiones relativas a la notación del controlador empleado para el guiado del robot. Se considera $\mathbf{q} \in \Re^{\mathrm{nx} 1}$ las coordenadas articulares del robot, $\dot{\mathbf{q}} \in \mathfrak{R}^{\mathrm{nx} 1}$ representan las velocidades articulares mientras que $\ddot{\mathbf{q}} \in \mathfrak{R}^{\mathrm{nx1}}$ son las aceleraciones articulares (n representa los grados de libertad o número de articulaciones del robot). Como se ha indicado anteriormente, el robot es guiado por información visual. En un caso general, esta información visual es un vector de $\mathrm{k}$ puntos en el espacio imagen, que se representan con el vector $\mathbf{s}=$ $\left[f_{1 x}, f_{1 y}, f_{2 x}, f_{2 y}, \ldots, f_{k x}, f_{k y}\right]^{\mathrm{T}} \in \mathfrak{R}^{2 \mathrm{k}}$. La matriz de interacción establece la siguiente relación entre el espacio imagen y el espacio Cartesiano 3D:

$$
\dot{\mathbf{s}}=\mathbf{L}_{\mathrm{s}}(\mathbf{s}, \mathbf{Z}) \dot{\mathbf{r}}
$$

donde $\dot{\mathbf{s}}$ es la derivada con respecto al tiempo de las características visuales extraídas, $\mathbf{r}$ representa la localización 3D del sistema de coordenadas asociado a la cámara del extremo del robot y $\dot{\mathbf{r}}$ es la velocidad correspondiente a la cámara. Se va a considerar que el robot se encuentra realizando el seguimiento en un espacio m-dimensional (por lo tanto, $\mathbf{r} \in \mathfrak{R}^{\mathrm{m}}$ ), de forma que si $n>m$ el robot será redundante. Dado que se considera un control visual con cámara montada en el extremo o eye-in-hand, la relación entre la cámara y el extremo del robot es fija y conocida. De ahí que se pueda hablar de $\mathbf{r}$ o $\mathbf{r}_{\mathrm{e}}$ indistintamente.

Por otro lado, la Jacobiana del robot relaciona la velocidad del efector final del robot y su velocidad articular, $\dot{\mathbf{q}}: \dot{\mathbf{r}}=\mathrm{J}_{\mathrm{r}}(\mathbf{q}) \dot{\mathbf{q}}$

$$
\dot{\mathbf{r}}=\mathrm{J}_{\mathrm{r}}(\mathbf{q}) \dot{\mathbf{q}}
$$

Haciendo uso de la Ecuaciones ¡Error! No se encuentra el origen de la referencia. y $i$ Error! No se encuentra el origen de la referencia. es posible obtener la siguiente ecuación que representa la relación entre la velocidad articular del robot y la variación con respecto al tiempo de las características visuales:

$$
\dot{\mathbf{s}}=\mathbf{L}_{s}(\mathbf{s}, \mathbf{Z}) \mathbf{J}_{\mathbf{r}}(\mathbf{q}) \dot{\mathbf{q}}=\mathbf{L}_{\mathrm{J}}(\mathbf{q}, \mathbf{s}, \mathbf{Z}) \dot{\mathbf{q}}
$$

donde $\mathbf{L}_{\mathrm{J}}=\mathbf{L}_{s}(\boldsymbol{r}) \mathbf{J}_{\mathbf{r}}(\mathbf{q})=\mathbf{L}_{\mathrm{J}}(\mathbf{q}, \mathbf{s}, \mathbf{Z}) \in \mathfrak{R}^{2 \mathrm{kxn}}$.

Derivando la Ecuación ¡Error! No se encuentra el origen de la referencia. con respecto al tiempo se puede obtener la siguiente expresión (con el objetivo de obtener una notación más compacta en las siguientes ecuaciones, no se indica la dependencia de las distintas Jacobianas con respecto a las variables articulares):

$$
\ddot{\mathbf{s}}=\mathbf{L}_{\mathrm{J}} \ddot{\mathbf{q}}_{\mathrm{r}}+\dot{\mathbf{L}}_{\mathrm{J}} \dot{\mathbf{q}}
$$

donde $\ddot{\mathbf{s}}$ representa la aceleración en el espacio imagen o segunda derivada con respecto al tiempo de las características visuales extraídas. Con el objetivo de permitir realizar el correcto seguimiento de una trayectoria especificada en el espacio imagen, se va a proponer un controlador basado en aceleración en el espacio articular. Es habitual encontrar en la bibliografía aproximaciones basadas en velocidad, debido principalmente a su simplicidad. Sin embargo, la aproximación basada en aceleración es más adecuada cuando se tiene en cuenta la dinámica del robot empleado en el control. Así, con $\ddot{\mathbf{q}}_{\mathrm{r}}$ se representa la aceleración articular que será aplicada al robot y generada por el controlador con el objetivo anteriormente comentado. 
La acción de control o aceleraciones articulares requeridas, $\ddot{\mathbf{q}}_{\mathrm{r}}$, pueden ser obtenidas a partir de ¡Error! No se encuentra el origen de la referencia.:

$$
\ddot{\mathbf{q}}_{\mathrm{r}}=\mathbf{L}_{\mathrm{J}}^{+}\left(\ddot{\mathbf{s}}_{\mathrm{r}}-\dot{\mathbf{L}}_{\mathrm{J}} \dot{\mathbf{q}}\right)
$$

Queda por definir la referencia en el espacio imagen, $\ddot{\mathbf{s}}_{\mathrm{r}}$, que permita desarrollar correctamente el seguimiento de las características visuales. Para ello se considera:

$$
\begin{gathered}
\ddot{\mathbf{s}}_{\mathrm{r}}=\ddot{\mathbf{s}}_{\mathrm{d}}+\mathbf{K}_{\mathrm{D}}\left(\dot{\mathbf{s}}_{\mathrm{d}}-\dot{\mathbf{s}}\right)+\mathbf{K}_{\mathrm{P}}\left(\mathbf{s}_{\mathrm{d}}-\mathbf{s}\right)= \\
\ddot{\mathbf{s}}_{\mathrm{d}}+\mathbf{K}_{\mathrm{D}} \dot{\mathbf{e}}_{\mathrm{s}}+\mathbf{K}_{\mathrm{P}} \mathbf{e}_{\mathrm{s}}
\end{gathered}
$$

donde $\ddot{\mathbf{s}}_{\mathrm{d}}, \dot{\mathbf{s}}_{\mathrm{d}}$ y $\mathbf{s}_{\mathrm{d}}$ son las aceleraciones, velocidades $\mathrm{y}$ posiciones deseadas para las características en la imagen. $\mathbf{K}_{\mathrm{p}}$ y $\mathbf{K}_{\mathrm{D}}$ son matrices $\mathrm{PD}$ de ganancias. Además, $\mathbf{e}_{\mathrm{s}}$, es el error en la imagen, y $\dot{\mathbf{e}}_{\mathrm{s}}$ es la derivada con respecto al tiempo del error en el espacio imagen.

\subsection{CONTROL VISUAL DIRECTO BASADO EN ACELERACIÓN}

A continuación, se extenderá el sistema de control visual para generar directamente los pares articulares necesarios para realizar el seguimiento. Para ello, se parte del modelo dinámico del robot:

$$
\boldsymbol{\tau}=\mathbf{M}(\mathbf{q}) \ddot{\mathbf{q}}_{\mathrm{r}}+\mathbf{C}(\mathbf{q}, \dot{\mathbf{q}})+\mathbf{g}(\mathbf{q})
$$

donde $\mathbf{M}(\mathbf{q}) \in \mathfrak{R}^{\text {nxn }}$ es la matriz de inercia del manipulador (definida positiva y simétrica), $\mathbf{C}(\mathbf{q}, \dot{\mathbf{q}}) \in$ $\mathfrak{R}^{\mathrm{nx} 1}$ es el vector de los pares centrípetos y de Coriolis, $\mathbf{g}(\mathbf{q})$ es la fuerza gravitacional. Finalmente, $\boldsymbol{\tau} \in \mathfrak{R}^{\mathrm{nx} 1}$ es el vector de los pares aplicados a las articulaciones. A partir de la utilización de las Ecuaciones ¡Error! No se encuentra el origen de la referencia., (1) y (2), el controlador visual directo que se obtiene será el que se representa en la siguiente ecuación:

$$
\begin{aligned}
\mathbf{\tau}=\mathbf{M}(\mathbf{q}) \mathbf{L}_{\mathrm{J}}^{+} & \left(\ddot{\mathbf{s}}_{\mathrm{d}}+\mathbf{K}_{\mathrm{D}} \dot{\mathbf{e}}_{\mathrm{s}}+\mathbf{K}_{\mathrm{P}} \mathbf{e}_{\mathrm{s}}-\dot{\mathbf{L}}_{\mathrm{J}} \dot{\mathbf{q}}\right)+ \\
& +\mathbf{C}(\mathbf{q}, \dot{\mathbf{q}})+\mathbf{g}(\mathbf{q})
\end{aligned}
$$

Teniendo en cuenta las hipótesis previas, la obtención del lazo cerrado se puede obtener de la siguiente manera:

$$
\mathbf{M}(\mathbf{q}) \ddot{\mathbf{q}}_{\mathrm{r}}=\mathbf{M}(\mathbf{q}) \mathbf{L}_{\mathrm{J}}^{+}\left(\ddot{\mathbf{s}}_{\mathrm{d}}+\mathbf{K}_{\mathrm{D}} \dot{\mathbf{e}}_{\mathrm{s}}+\mathbf{K}_{\mathrm{P}} \mathbf{e}_{\mathrm{s}}-\dot{\mathbf{L}}_{\mathrm{J}} \dot{\mathbf{q}}\right)
$$

Realizando la pre-multiplicación de $\mathbf{L}_{\mathrm{J}}(\mathbf{M}(\mathbf{q}))^{\mathbf{- 1}}$ en ambos lados de la ecuación (4):

$$
\mathbf{L}_{\mathrm{J}} \ddot{\mathbf{q}}_{\mathrm{r}}=\ddot{\mathbf{s}}_{\mathrm{r}}-\dot{\mathbf{L}}_{\mathrm{J}} \dot{\mathbf{q}} \rightarrow \mathbf{L}_{\mathrm{J}} \ddot{\mathbf{q}}_{\mathrm{r}}+\dot{\mathbf{L}}_{\mathrm{J}} \dot{\mathbf{q}}=\ddot{\mathbf{s}}_{\mathrm{r}}
$$

Tomando en consideración la ecuación ¡Error! No se encuentra el origen de la referencia., a la parte izquierda de la ecuación previa se le llamará $\ddot{\mathbf{s}}$ y utilizando la ecuación (1), la parte derecha será $\ddot{\mathbf{s}}_{\mathrm{d}}+$ $\mathbf{K}_{\mathrm{D}} \dot{\mathbf{e}}_{\mathrm{s}}+\mathbf{K}_{\mathrm{P}} \mathbf{e}_{\mathrm{s}}$. Por lo tanto:

$$
\ddot{\mathbf{e}}_{\mathrm{s}}+\mathbf{K}_{\mathrm{D}} \dot{\mathbf{e}}_{\mathrm{s}}+\mathbf{K}_{\mathrm{P}} \mathbf{e}_{\mathrm{s}}=0
$$

Con la ecuación anterior se consigue un seguimiento asintótico en el espacio imagen.

\section{OPTIMIZACIÓN EN CONTROL VISUAL}

La función de control que minimiza los pares aplicados a un robot manipulador mientras se ejecuta una tarea de control visual determinada viene definida por:

$$
\begin{gathered}
\mathbf{\tau}=\mathbf{W}^{-1 / 2}\left(\mathbf{A} \mathbf{M}^{-1} \mathbf{W}^{-1 / 2}\right)^{+} \\
\cdot\left(\mathbf{b}-\mathbf{A} \mathbf{M}^{-1}(-\mathbf{C}-\mathbf{g})\right)
\end{gathered}
$$

donde $\mathbf{W}$ es una matriz de pesos dependiente del tiempo y las $\mathrm{m}$ restricciones de la tarea que se debe ejecutar vienen dadas por:

$$
\mathbf{A}(\mathbf{q}, \dot{\mathbf{q}}, \mathrm{t}) \ddot{\mathbf{q}}=\mathbf{b}(\mathbf{q}, \dot{\mathbf{q}}, \mathrm{t})
$$

donde $\mathbf{A}=\mathbf{A}(\mathbf{q}, \dot{\mathbf{q}}, \mathrm{t}) \in \mathfrak{R}^{m x n}$ y $\mathbf{b}=\mathbf{b}(\mathbf{q}, \dot{\mathbf{q}}, \mathrm{t}) \in$ $\mathfrak{R}^{m x 1}$.

El principal objetivo a alcanzar con los controladores que se quieren derivar de este framework es guiar al robot a lo largo de una trayectoria deseada en el espacio imagen. En este caso, la descripción de la tarea viene determinada por la siguiente ecuación en el espacio imagen:

$$
\left(\ddot{\mathbf{s}}_{\mathrm{d}}-\ddot{\mathbf{s}}\right)+\mathbf{K}_{\mathrm{D}}\left(\dot{\mathbf{s}}_{\mathrm{d}}-\dot{\mathbf{s}}\right)+\mathbf{K}_{\mathrm{P}}\left(\mathbf{s}_{\mathrm{d}}-\mathbf{s}\right)=0
$$

donde $\mathbf{K}_{\mathrm{P}}$ y $\mathbf{K}_{\mathrm{D}}$ son las ganancias proporcional y derivativa, respectivamente. Esta ecuación se puede expresar con respecto al error en la imagen según la ecuación (1). A partir de (1) y (5) se puede obtener:

$$
\ddot{\mathbf{s}}_{\mathrm{d}}+\mathbf{K}_{\mathrm{D}} \dot{\mathbf{e}}_{\mathrm{s}}+\mathbf{K}_{\mathrm{P}} \mathbf{e}_{\mathrm{s}}-\dot{\mathbf{L}}_{\mathrm{J}} \dot{\mathbf{q}}=\mathbf{L}_{\mathrm{J}} \ddot{\mathbf{q}}
$$

Ahora es posible expresar las restricciones de la tarea de la ecuación (10) en la forma de (8) con:

$$
\begin{gathered}
\mathbf{A}=\mathbf{L}_{\mathrm{J}} \\
\mathbf{b}=\ddot{\mathbf{s}}_{\mathrm{d}}+\mathbf{K}_{\mathrm{D}} \dot{\mathbf{e}}_{\mathrm{s}}+\mathbf{K}_{\mathrm{P}} \mathbf{e}_{\mathrm{s}}-\dot{\mathbf{L}}_{\mathrm{J}} \dot{\mathbf{q}}
\end{gathered}
$$

Reemplazando las variables concernientes a la descripción de la tarea de imagen, A y b de (11), la ley de control es la siguiente:

$$
\begin{gathered}
\mathbf{\tau}=\mathbf{W}^{-1 / 2}\left(\mathbf{L}_{\mathrm{J}} \mathbf{M}^{-1} \mathbf{W}^{-1 / 2}\right)^{+} . \\
\cdot\left(\ddot{\mathbf{s}}_{\mathrm{d}}+\mathbf{K}_{\mathrm{D}} \dot{\mathbf{e}}_{\mathrm{s}}+\mathbf{K}_{\mathrm{P}} \mathbf{e}_{\mathrm{s}}-\dot{\mathbf{L}}_{\mathrm{J}} \dot{\mathbf{q}}-\right. \\
\left.-\mathbf{L}_{\mathrm{J}} \mathbf{M}^{-1}(-\mathbf{C}-\mathbf{g})\right)
\end{gathered}
$$


En el siguiente apartado se describirán los resultados y simulaciones obtenidas en la aplicación de este controlador.

\section{RESULTADOS}

Esta sección presenta diferentes experimentos con el objetivo de ilustrar el comportamiento del sistema de control visual propuesto. Los sistemas de control visual propuesto se aplican a un robot planar RRR con ejes paralelos y con la cámara en su extremo mirando hacia un plano 2D paralelo. Sus articulaciones permiten el posicionamiento del extremo (o de la cámara, ya que la relación de los sistemas de referencia de ésta con respecto al efector final será fija y conocida) en ese plano paralelo al robot. Así, los experimentos de control se centran en posicionamientos y seguimiento de trayectorias en el plano XY.

Los parámetros intrínsecos considerados para la cámara son $\left(\mathrm{u}_{0}, \mathrm{v}_{0}\right)=(368,290) \mathrm{px}, \mathrm{y}\left(\mathrm{f}_{\mathrm{u}}, \mathrm{f}_{\mathrm{v}}\right)=$ $(433,433)$ px (posición del centro óptico $\left(\mathrm{u}_{0}, \mathrm{v}_{0}\right)$ y la longitud focal en las direcciones $\mathrm{X}$ e $\mathrm{Y}$ respectivamente).

\subsection{CONTROL VISUAL DIRECTO BASADO EN PAR CALCULADO}

Este experimento evalúa la implementación del controlador propuesto en (3) para el seguimiento de una trayectoria en imagen repetitiva. Dicha trayectoria deseada para la característica extraída en la imagen viene definida por la siguiente ecuación:

$$
\begin{gathered}
\boldsymbol{s}_{d}=\left[\begin{array}{l}
f_{x d} \\
f_{y d}
\end{array}\right] \\
f_{x d}=320+166 \cos (\omega t+\pi / 4)+ \\
+20 \sin (\omega 5 t) \\
f_{y d}=265+160 \cos (\omega t+\pi / 4)+ \\
+15 \sin (\omega 5 t)
\end{gathered}
$$

En la Figura 1 se representa en rojo la trayectoria deseada en el espacio de la imagen definida haciendo uso de la Ecuación (13). En esta figura se observa en azul el comportamiento obtenido durante el seguimiento considerando una velocidad media/alta $(\omega=1 \mathrm{rad} / \mathrm{s})$. En este experimento se han considerado los siguientes valores para las ganancias del controlador $\mathbf{K}_{\mathrm{P}}=0.1 \mathrm{I}$ y $\mathbf{K}_{\mathrm{D}}=0.5 \mathrm{I}$ (ganancias proporcional y derivativa para la trayectoria deseada a seguir). Se observa que se obtiene un correcto comportamiento y los errores de seguimiento son mínimos. Por último, en la Figura 2 se representan los pares articulares aplicados por el robot y que permiten llevar a cabo correctamente el seguimiento.

Con el objetivo de evaluar el comportamiento del controlador implementado cuando se aplican distintas velocidades de seguimiento, la Tabla 1 representa el error medio en píxeles cuando el parámetro $\omega$ de la Ecuación (3) vale $0.5,1$ y $2 \mathrm{rad} / \mathrm{s}$, respectivamente.

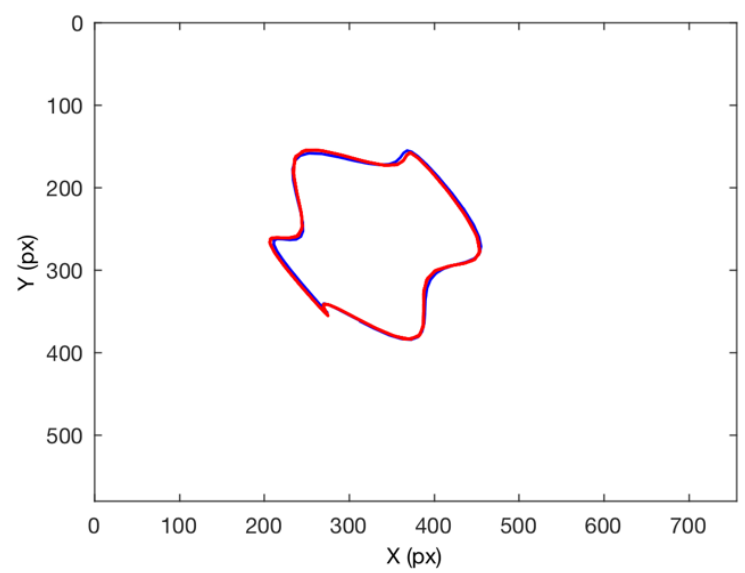

Figura 1: Trayectoria deseada y obtenida en la imagen con el controlador visual basado en par calculado
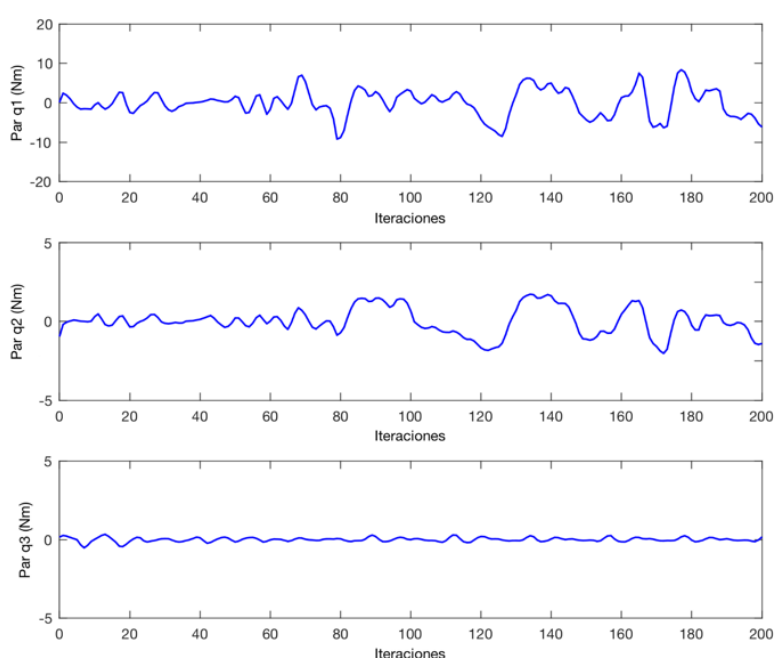

Figura 2: Par articular aplicado por el controlador directo basado en par calculado

Tabla 1: Error medio en píxeles considerando distintas velocidades de seguimiento.

\begin{tabular}{|c|c|c|}
\hline $\boldsymbol{\omega}=\mathbf{0 . 5} \mathbf{~ r a d} / \mathbf{s}$ & $\boldsymbol{\omega}=\mathbf{1 ~} \mathbf{~ a d} / \mathbf{s}$ & $\boldsymbol{\omega}=\mathbf{2} \mathbf{~ r a d} / \mathbf{s}$ \\
\hline 2.14 & 2.55 & 3.1 \\
\hline
\end{tabular}




\subsection{CONTROL VISUAL DIRECTO CON OPTIMIZACIÓN}

Como se observa en la Ecuación (12), el controlador propuesto basado en control óptimo para el seguimiento de trayectorias depende de la matriz $\mathbf{W}$. Esta matriz determina cómo se distribuyen los pares articulares en las articulaciones. Considerando diferentes valores para esta matriz, se pueden obtener controladores visuales directos basados en imagen conocidos, al tiempo que se pueden obtener nuevos controladores con comportamientos dinámicos distintos. Por ejemplo, si se elige $\mathbf{W}=\mathbf{M}^{-2}$, se obtiene el controlador propuesto en (3). Otros valores interesantes para la matriz $\mathbf{W}$ son I y $\mathbf{D}$, siendo I la matriz identidad y $\mathbf{D}$ una matriz diagonal positiva. Al elegir la matriz I se simplifica la ley de control:

$$
\begin{gathered}
\mathbf{\tau}=\left(\mathbf{L}_{\mathrm{J}} \mathbf{M}^{-1}\right)^{+} \cdot \\
\cdot\left(\ddot{\mathbf{s}}_{\mathrm{d}}+\mathbf{K}_{\mathrm{D}} \dot{\mathbf{e}}_{\mathrm{s}}+\mathbf{K}_{\mathrm{P}} \mathbf{e}_{\mathrm{s}}-\dot{\mathbf{L}}_{\mathrm{J}} \dot{\mathbf{q}}-\right. \\
\left.-\mathbf{L}_{\mathrm{J}} \mathbf{M}^{-1}(-\mathbf{C}-\mathbf{g})\right)+\boldsymbol{\tau}_{\mathrm{D}}
\end{gathered}
$$

Eligiendo $\mathbf{W}=\mathbf{D}$, se permite distribuir los pares en las articulaciones, de forma que pesos grandes causarán pares pequeños en las articulaciones correspondientes:

$$
\begin{gathered}
\boldsymbol{\tau}=\mathbf{D}^{-1 / 2}\left(\mathbf{L}_{\mathrm{J}} \mathbf{M}^{-1} \mathbf{D}^{-1 / 2}\right)^{+} . \\
\cdot\left(\ddot{\mathbf{s}}_{\mathrm{d}}+\mathbf{K}_{\mathrm{D}} \dot{\mathbf{e}}_{\mathrm{s}}+\mathbf{K}_{\mathrm{P}} \mathbf{e}_{\mathrm{s}}-\dot{\mathbf{L}}_{\mathrm{J}} \dot{\mathbf{q}}-\right. \\
\left.-\mathbf{L}_{\mathrm{J}} \mathbf{M}^{-1}(-\mathbf{C}-\mathbf{g})\right)+\boldsymbol{\tau}_{\mathrm{DFC}}
\end{gathered}
$$

Como se describe en [12], otro valor que resulta interesante considerar para $\mathbf{W}$ es $\mathbf{M}^{-1}$, ya que es consistente con el principio de d'Alembert. La ley de control obtenida en este caso es igual a:

$$
\begin{gathered}
\boldsymbol{\tau}=\mathbf{M}^{1 / 2}\left(\mathbf{L}_{\mathrm{J}} \mathbf{M}^{-1} \mathbf{M}^{1 / 2}\right)^{+} \cdot \\
\cdot\left(\ddot{\mathbf{s}}_{\mathrm{d}}+\mathbf{K}_{\mathrm{D}} \dot{\mathbf{e}}_{\mathrm{s}}+\mathbf{K}_{\mathrm{P}} \mathbf{e}_{\mathrm{s}}-\dot{\mathbf{L}}_{\mathrm{J}} \dot{\mathbf{q}}-\right. \\
\left.-\mathbf{L}_{\mathrm{J}} \mathbf{M}^{-1}(-\mathbf{C}-\mathbf{g})\right)+\boldsymbol{\tau}_{\mathrm{DFC}}
\end{gathered}
$$

Considerando los valores indicados previamente para $\mathbf{W}$, se ha realizado el mismo experimento de seguimiento de trayectoria en el espacio imagen descrito por la Ecuación (13). Cabe mencionar que otros valores de $\mathbf{W}$ pueden proporcionar al controlador obtenido diferentes propiedades dinámicas durante el seguimiento de la trayectoria en la imagen.

Como muestra la Figura 3, se obtiene un correcto seguimiento de la trayectoria deseada en todos los casos cuando se emplea una velocidad alta $(\omega=1$ $\mathrm{rad} / \mathrm{s}$ ). El mejor comportamiento lo proporciona $\mathbf{W}=\mathbf{M}^{-1}$, aunque el seguimiento de la trayectoria es similar al obtenido con el controlador propuesto en (3). En todos los experimentos mostrados en este apartado, la ganancia proporcional ha sido establecida en $\mathbf{K}_{\mathrm{P}}=0.1 \mathbf{I}$, y la ganancia derivativa en $\mathbf{K}_{\mathrm{D}}=0.5 \mathbf{I}$. Dado que es complicado distinguir en las gráficas de la Figura 3 el comportamiento de uno y otro controlador, en la Figura 4 se muestran las acciones de control o pares generados durante el experimento. La matriz $\mathbf{D}$ es una matriz diagonal donde el peso correspondiente a la tercera articulación se ha establecido al doble del peso correspondiente a la primera y la segunda articulaciones. Se observa en la Figura 3 el efecto que se obtiene con esta matriz. Si comparamos la Figura 3.b con la Figura 3.c se puede observar que cuando $\mathbf{W}=\mathbf{D}$ se obtienen pares más pequeños en la tercera articulación (tal como se esperaba, ya que el peso correspondiente a esta articulación era dos veces mayor que los pesos de las otras dos articulaciones). Así, esta matriz diagonal se puede emplear para distribuir los pares y disminuir el esfuerzo en las articulaciones que se desee.

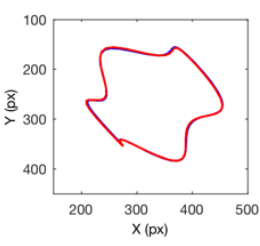

(a)

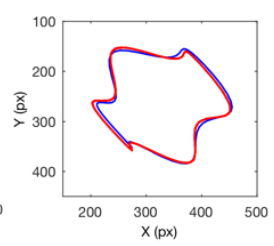

(b)

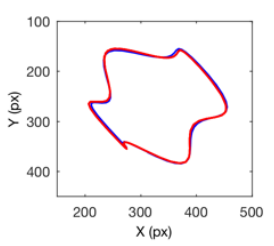

(c)
Figura 3: Trayectoria deseada en la imagen (azul) y trayectoria obtenida (rojo): (a) $\mathbf{W}=\mathbf{M}^{-1}$; (b) $\mathbf{W}=\mathbf{D}$;

(c) $\mathbf{W}=\mathbf{I}$

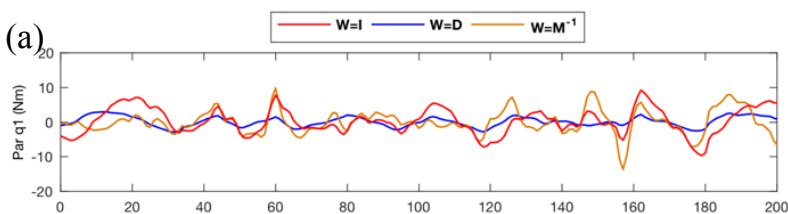

(b)
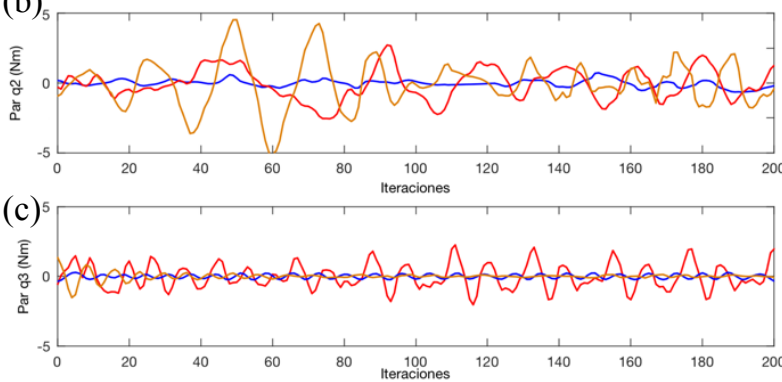

Figura 4: Pares articulares durante el experimento: W $=\mathbf{M}^{-1}($ azul $) ; \mathbf{W}=\mathbf{D}$ (naranja) $\mathbf{W}=\mathbf{I}($ rojo $)$.

\section{CONCLUSIONES}

En este artículo se realiza una comparativa de un sistema de control visual directo para el seguimiento de trayectorias basado en par calculado frente al uso 
de un controlador visual basado en optimización. Como se puede extraer de los resultados obtenidos, el controlador basado en par calculado puede considerarse como un caso específico del controlador basado en optimización. De hecho, el primero puede obtenerse para un valor específico de la matriz de pesos $\left(\mathbf{W}=\mathbf{M}^{-2}\right)$. En el artículo se muestran los últimos resultados obtenidos en simulación y actualmente se está realizando la implementación de los mismos en un manipulador redundante de 7 grados de libertad.

\section{English summary}

\section{OPTIMIZATION IN VISUAL SERVOING IN ROBOT MANIPULATORS}

\section{Abstract}

This paper describes the formulation of a direct imagebased visual servoing system for tracking trajectories with optimization of joint effort. The main components of this control strategy are described for its application to the guidance of manipulators. Making use of this control law it is possible, not only to optimize the torques to be applied for tracking trajectories, but also to specify the joints that will support more or less effort during the tracking. After describing the formulation, the validity of the controllers is demonstrated by simulation of a robot with three degrees of freedom.
\end{abstract}

Keywords: visual servoing, robot control, optimal control.

\section{Agradecimientos}

Este trabajo ha sido financiado por el Ministerio de Economía, Industria y Competitividad de España a través del proyecto DPI2015-68087-R así como por los Fondos FEDER.

\section{Referencias}

[1] F. Chaumette, and S. Hutchinson, (2006) "Visual Servo Control, Part I: Basic Approaches". IEEE Robotics and Automation Magazine, vol. 13, no. 4, pp. 82-90.

[2] C. C. Cheah, S. P. Hou, Y. Zhao, y J. J. E. Slotine, (2010) "Adaptive vision and force tracking control for robots with constraint uncertainty". IEEE/ASME Transactions on Mechatronics, vol. 15, no. 3, pp. 389-399.

[3] C. C. Cheah, S. P. Hou, Y. Zhao, y J. J. E. Slotine, (2007) "Adaptive Vision based Tracking Control of Robots with Uncertainty in Depth
Information". IEEE International Conference on Robotics and Automation, Roma, pp. 2817-2822.

[4] G. Fink, H. Xie, A. F. Lynch, M. Jagersand (2015). "Experimental validation of dynamic visual servoing for a quadrotor using a virtual camera" International Conference on Unmanned Aircraft Systems, pp. 1231-1240.

[5] A. H. Jabbari, G. Oriolo, H. Bolandi, (2014) "Output feedback image-based visual servoing control of an underactuated unmanned aerial vehicle" Proceedings of the Institution of Mechanical Engineers. Part I: Journal of Systems and Control Engineering, vol. 228, no. 7, pp. 435448.

[6] C. A. Jara, J Pomares, F.A. Candelas, F. Torres, (2014) "Control framework for dexterous manipulation using dynamic visual servoing and tactile sensors' feedback" Sensors, vol. 14, no. 1, pp. 1787-1804.

[7] R. Kelly, R. Carelli, O. Nasisi, B. Kuchen, and F. Reyes, (2000) "Stable Visual Servoing of Camera-in-Hand Robotic Systems". IEEE/ASME Transactions on Mechatronics, vol. 5, no. 1, pp. 39-48.

[8] X. Li, C. C. Cheah, (2013) "Global task-space adaptive control of robot" Automatica, vol. 49, no. 1, pp. 58-69.

[9] R. Mahony, (2017). "Modular design of image based visual servo control for dynamic mechanical systems" Springer Tracts in Advanced Robotics, vol. 100, pp. 129-146.

[10] J. Pomares, J. A. Corrales, G. J. García, F. Torres, (2011) "Direct visual Servoing to track trajectories in human-robot cooperation". International Journal of Advanced Robotic Systems, vol. 8, no. 4, pp. 129-138.

[11]P. Serra, R. Cunha, T. Hamel, D. Cabecinhas, C. Silvestre, (2016) "Landing of a quadrotor on a moving target using dynamic image-based visual servo control" IEEE Transactions on Robotics, vol. 32, no. 6, pp. 1524-1535.

[12]F. E. Udwadia, (2003) "A new perspective on tracking control of nonlinear structural and mechanical systems", Proc. of the Royal Society of London Series A, pp. 1783-1800.

[13]H. Wang, W. Chen, C. Wang, X. Wang, R. Pfeifer, (2014) "Dynamic modeling and imagebased adaptive visual servoing of cable-driven soft robotic manipulator" IFAC Proceedings Volumes, vol. 19, pp. 11884-11889.

[14]Xie, H., Lynch, A.F., Jagersand, M. (2016) "Dynamic IBVS of a rotary wing UAV using line features", Robotica, vol. 34, no. 9, pp. 20092026. 
(C) 2018 by the authors. Submitted for possible open access publication under the terms and conditions of the Creative Commons Attribution CC-BY-NC 3.0 license (https://creativecommons.org/licenses/by-nc/3.0). 